\title{
Una aproximación conceptual a la cualificación profesional desde una perspectiva competencial
}

\author{
Ángel Olaz \\ Departamento de Sociología y Política Social. Facultad de Economía y Empresa. \\ Campus Universitario de Espinardo. Murcia \\ olazcapi@um.es
}

Aceptado: 02-07-2010

\section{Resumen}

El estudio de la cualificación profesional es un tema recurrente en la sociología por parte de diferentes escuelas y autores. En primer lugar, este trabajo tiene el propósito inicial de realizar una revisión histórica de su significado y, en segundo lugar, de vincular este concepto con el de competencia, proponiendo una redefinición del término cualificación como variable dependiente de las competencias. Por último, este trabajo propone una nueva definición del término competencia basado en la interacción de los conocimientos, capacidades y habilidades.

Palabras clave: capital humano; conocimientos; competencia; cualificación; habilidades.

Abstract. A conceptual approximation to the professional qualification from the perspective of competence

The study of professional qualification is a recurring matter in Sociology among different schools and authors. First of all, this paper aims to carry out an initial review of his historical significance and secondly, link this concept with competence, proposing a redefinition of the qualification as a dependent variable of competences. Finally this paper proposes a new definition of competence based on the interaction of knowledges, capabilities and abilities.

Key words: human capital; knowledges; competence; qualification; skills.

\section{Sumario}

\section{Introducción}

1. Una aproximación al concepto de cualificación profesional

2. Las competencias

3. Competencias y cualificación
4. Hacia una definción del término competencia

5. Conclusiones

Bibliografía 


\section{Introducción}

Tradicionalmente, el estudio de la cualificación se ha considerado un tema controvertido en el terreno de las ciencias sociales y, de manera especial, en la sociología. Diferentes escuelas de pensamiento y autores han aportado sus reflexiones acerca de cuál es su significado y de qué modo puede entenderse, sin que, finalmente, el debate haya cesado, incluso al contrario, han surgido nuevos enfoques que permiten sondear el contenido, el alcance y la dimensión que la cualificación profesional del trabajador tiene en primera persona, con respecto al grupo de trabajo, la organización en la que desarrolla su actividad y la sociedad de la que forma parte.

Lejos de plantear una discusión teórica acerca de cuál es la verdadera esencia y significado del concepto cualificación, este trabajo plantea abordar la caracterización de este término a través del significado que en ella tiene el término competencia.

En esta línea de análisis, la competencia es, asimismo, construida como aquel conjunto de conocimientos, capacidades y habilidades, modeladas por el carácter individual o grupal de quien las pone en práctica y, en última instancia, la gestiona.

Desde este punto de vista, son las competencias las que, de algún modo, se convierten en variables explicativas de la cualificación, de igual modo que la cualificación no podría entenderse sin el referente competencial.

Este escenario conceptual, académico y profesional no por ello podría negar la existencia de desajustes como la sobrecualificación o la infracualificación del individuo en el puesto de trabajo, resultado del desequilibrio entre los niveles de cualificación y competenciales.

En suma, un controvertido escenario donde la propia realidad social modela y construye un conjunto de situaciones y comportamientos abierto a la especulación y a la intervención sociológica en un contexto de rápida aceleración histórica.

\section{Una aproximación al concepto de cualificación profesional}

Cuando la definición de un término no es posible realizarla de forma inmediata, es recomendable acercarse a él a través de aproximaciones sucesivas. Sucede que la cualificación laboral, más allá de conocimientos y prácticas aplicadas a las tareas que uno realiza, es esencialmente expresión de la persona y de su relación con los demás, y queda manifestada a través de la actuación cotidiana en el trabajo, sin olvidar el peso de la construcción social que en ella interviene. Según Castillo (1996: 90):

Si la cualificación es socialmente construida, especialmente, en la gran empresa, obviamente, en los sistemas de empresas, formados por muchas pequeñas empresas (e incluso grandes), dispersas en un territorio, y, por tanto, [más] abiertas a la influencia "externa» e institucional, esta construcción social aparecerá y se manifestará, resaltará de manera más clara y diáfana. 
Aparte de su construcción social, es importante contemplar tres aspectos que, en opinión de este autor, deben mencionarse; por una parte, la cualificación «dentro de» y como resultado de una determinada división del trabajo, con independencia del sector considerado, debido — este es el segundo elemento- - a que la inteligencia global aplicada a la producción está cada vez más institucionalizada, y, por último, porque el evolucionar de las cualificaciones depende de la división, de ese saber entre los trabajadores y las instituciones que componen la sociedad.

Se trata de descomponer este concepto en una imagen caleidoscópica, en la que contemplamos la cualificación propia del trabajador, resultado de su propio bagaje intelectual, junto a la que requiere el puesto de trabajo donde ha de desarrollar su función, pasando por los requerimientos institucionales y de mercado.

El concepto de cualificación que se adopta en este trabajo tomado como punto de partida nace del conocimiento previo del proceso laboral, ya que donde la cualificación puede analizarse de forma más precisa es el lugar en que los trabajadores realizan sus funciones, esto es, en el puesto de trabajo.

La fundamentación de esta hipótesis se basa en dos cuestiones, por un lado, es en el puesto de trabajo donde los asalariados llevan a cabo sus tareas. Para ello, aplican las capacidades que esas tareas exigen y, por otro, las características de cada uno de los puestos están definidas por la estructura del proceso de trabajo en que se insertan.

\subsection{Acepciones del término cualificación comúnmente aceptadas}

Un breve repaso a algunas conceptualizaciones del concepto de cualificación profesional nos lleva, sin embargo, a tomar buena nota de las principales aportaciones que diferentes autores han hecho sobre este particular, especialmente durante las décadas de 1980 y 1990, que es donde mayor protagonismo adquirió la discusión del término.

Petroni (1987) equipara el concepto de profesionalidad al de cualificación y, según él, está constituida por las siguientes dimensiones: el contenido del trabajo, el empeño físico y psíquico (puesto en marcha para desarrollar las tareas) y la cultura laboral, es decir, los valores y los comportamientos que lo acompañan. Contempla lo que Villavicencio (1992) denomina «dimensiones cognoscitivas» (para el desarrollo de las tareas) y «sociales» (los valores y los comportamientos).

Evans (1982) considera que la cualificación forma parte del contenido del trabajo, y éste, a su vez, de la calidad del trabajo, constituida, además, por los factores que conducen al significado del trabajo, el aprendizaje, el entorno en que se realiza, la seguridad laboral o la remuneración. Por otra parte, amplía este significado al indicar que del contenido del trabajo forman parte la cualificación, la responsabilidad y la libertad en la supervisión y el control sobre el ritmo laboral.

Existen también una serie de dimensiones, como la actividad intelectual o los conocimientos y su puesta en práctica en el desarrollo de las tareas, que son 
referencia inexcusable en el concepto de cualificación, según la definición de Bessant (1992).

Littler (1982) considera los conocimientos y las características de las rutinas de trabajo como factores constitutivos de la cualificación.

En otro orden de ideas, Sorge y Streeck (1993) aluden a los conocimientos adquiridos o necesarios para ejecutar las tareas $y$, aunque diferencian entre factores aptitudinales y organizacionales para referirse a los que inciden sobre las calificaciones, unos y otros se confunden entre sí en su análisis.

Por su parte, Coriat (1993) alude al conocimiento de los modos operativos del trabajo como Sabel (1986), el cual describe a la cualificación como la capacidad para ordenar a un equipo que realice las operaciones necesarias o, dicho de otro modo, la complejidad del trabajo como elemento que ayuda a describir la cualificación.

Sin embargo, en ocasiones, la cualificación se reduce a la categoría profesional. Esta última perspectiva es usual en autores como Boyer (1986a), Alaluf (1988), Leborgne y Lipietz (1988), que se incluyen en el enfoque regulacionista. Para ellos, la cualificación es fruto de las relaciones entre capital y trabajo y la analizan con términos de jerarquías profesionales y salariales, lo que restringe el alcance del concepto a lo que podría denominarse la «cualificación reconocida por la empresa».

Existen otros aspectos de la cualificación, como son: actividad intelectual, conocimientos técnicos y prácticos (habilidades), iniciativa, experiencia, autonomía, capacidad de intervención y complejidad del trabajo, los cuales están emparentados con las relaciones establecidas entre las personas que intervienen en el proceso de producción, así como entre ellas y los equipos productivos. Estas reflexiones aluden al «saber hacer», el llamado Know How al que aluden Barcet, Le Bas y Mercier (1985), según la terminología anglosajona, entendido como el conjunto de capacidades operacionales que derivan de su práctica productiva. De algún modo, se está haciendo referencia a la configuración específica del proceso de trabajo donde, finalmente, la cualificación de cada trabajador se articula en torno a las exigencias impuestas por las tareas; los medios de producción que utiliza y las relaciones que establece con ellos; y, por último, el modo de organizar el trabajo y de desempeñar las tareas.

Obviamente, la cualificación es una cuestión de dominio técnico, pero también es un problema de índole social. Depende de la interpretación que hacen las diferentes escuelas que han abordado su análisis. Un problema que remite al contexto histórico y cultural que está saturado de condicionamientos de género, como señala Cockburn (1985).

No obstante, y al margen de sus raíces sociales, estudiar la cualificación implica acercarse al lugar donde se concreta: la empresa, el proceso y el puesto de trabajo. Y de ahí que sus contenidos no se asocien necesariamente a la certificación normal de los conocimientos (a la titulación), y menos aún a realidades sociales como la española, donde existe una centralidad de la empresa, como señala Pries (1988). 
Freyssenet (1979) distingue entre siete tipos diferentes de cualificaciones:

a) Cualificación real requerida o cualificación efectiva, que es el conocimiento de las tareas según criterios del empresario.

b) Cualificación real del trabajador, que comprende dos subcategorías: b.1) cualificación real útil o conocimientos necesarios en el proceso productivo y que poseen los afectados, y b.2) otros conocimientos que no entran en el proceso de trabajo y que, por tanto, no son útiles en las tareas.

c) Cualificación atribuida oficialmente a los puestos de trabajo, asimilable a las categorías laborales, o, lo que es lo mismo, la que es reconocida oficialmente por la empresa.

d) Cualificación atribuida tras recibir una formación.

e) Cualificación exigida para ser contratado para un puesto de trabajo dado y que, en situaciones de paro elevado, presenta importantes distorsiones respecto a las tareas realizadas. Esta situación provoca la contratación de titulados superiores para puestos de escasa complejidad intelectual.

f) La suma de cualificaciones reales que supone un proceso de trabajo dado o la suma de conocimientos que es necesario aplicar en el conjunto del proceso de trabajo.

g) Cualificación atribuida por organismos estadísticos.

Por último, haciendo una lectura del concepto de cualificación, Castillo (1987: 388) menciona lo que algunos han llamado «calificación comportamental», entendida como:

[...] una serie de rasgos, no definidos con claridad, pero no específicamente comprendidos entre las calificaciones técnicas, y que integran el ser flexibles, disciplinados e identificados sobre todo con los objetivos de producción y calidad.

El análisis del proceso laboral pone en evidencia que los trabajadores adquieren una serie de capacidades de manera informal que les permiten atender - mejor que lo especificado por las normas de trabajo- las exigencias de sus tareas. Desde luego, esas habilidades, pequeños trucos o conocimientos específicos, en definitiva, se pueden adquirir individualmente.

Pero, como ya indicara Marx, los conocimientos adquiridos por la experiencia se acumulan en el colectivo de trabajadores y se transmiten entre ellos, lo cual indica de algún modo el concepto de cualificación laboral del trabajador asalariado. Es lo que podría denominarse el «boca a boca» de las estructuras informales.

En cualquier caso, la idea de cualificación no debe disociarse del mundo laboral. En este sentido, Castillo (2000: 40) indica que:

[...] la «cualificación profesional» es el resultado de la capacidad de trabajo de cada trabajador y del conocimiento del oficio o profesión. Entendido éste como la suma de habilidades individuales y colectivas y conocimientos del uso 
de herramientas y máquinas propias del oficio (saber hacer), y la tarea asignada dentro del proceso de trabajo (organización del trabajo).

Es por ello que, desde un punto de vista formal, la cualificación puede entenderse como el conjunto de competencias profesionales (conocimientos y capacidades) que permiten dar respuesta a ocupaciones y a puestos de trabajo con valor en el mercado laboral, y que pueden adquirirse a través de formación o de experiencia. En otras palabras, la cualificación profesional es el «conjunto de competencias profesionales con significación en el empleo que pueden ser adquiridas mediante formación modular $\mathrm{u}$ otros tipos de formación, así como a través de la experiencia laboral» (Ley 5/2002 de las cualificaciones y de la formación profesional). De forma más sintética, se entiende que una persona está cualificada cuando en su desempeño laboral obtiene los resultados esperados, con los recursos y el nivel de calidad debidos.

Cuestión aparte es el resultado final de este ambicioso proyecto, que algunos autores, como Roquero y Hernando (2004), señalan como un proceso inacabado.

\subsection{Escuelas de pensamiento sobre la cualificación profesional}

Es evidente que el monopolio del conocimiento de los profesionales, cada vez más está puesto en peligro por la acción combinada del nivel educativo de la población, el efecto de las nuevas tecnologías en el contexto de la sociedad red o sociedad de la información y la división del trabajo existente entre las distintas profesiones.

Básicamente, pueden encontrarse tres grandes enfoques epistemológicos. En primer término, debe mencionarse la corriente formada por los teóricos del capital humano, que señala como el uso de las nuevas tecnologías en sociedades avanzadas incrementa genéricamente el nivel de las cualificaciones.

En segundo lugar, otra escuela de pensamiento, en cuya cabeza figura Braverman, indica que la implantación de la tecnología y la automatización de los procesos de trabajo generan, a la larga, la pérdida de los saberes tradicionales de los trabajadores. Dicho en otras palabras: la descualificación.

Por último, la tercera postura, denominada «contingente», pondera cada una de las dos anteriores, con lo cual incide en cómo es posible encontrar tanto la calificación como la descualificación a la vez.

\subsubsection{Escuela del Capital Humano}

Como cada corriente teórica, la del capital humano nació en un momento histórico concreto. Fue durante la Administración Kennedy, cuando la elevación de las cualificaciones profesionales respondía al desarrollo expansionista de la economía norteamericana.

Era, pues, un momento en el que existía un enorme optimismo y una gran confianza sobre las ventajas que ofrecía la planificación para superar el atraso y el subdesarrollo social y económico. 
Este aparente buen momento, sin embargo, planteaba una curiosa paradoja, que señaló Bowman (1963: 446):

Nunca ha habido tanta educación, y, sin embargo, nunca se ha hablado tanto de la escasez de personas cualificadas o de la necesidad de expandir la educación como una inversión en capital humano.

Los trabajos centrados en el capital humano han subrayado, desde las obras pioneras de Schultz (1961) y Becker (1964), el concepto de inversión en educación. Bien es cierto que la educación reglada no es la única forma de capital humano, pero las dificultades para medir otras componentes del mismo (la salud, la formación no reglada o en el trabajo, etc.) han centrado la atención sobre la escolaridad.

Siguiendo el enfoque de la escuela, la rentabilidad de la formación desde el punto de vista individual termina siendo definitivo en el análisis, y concluye que la educación, además de constituir un impulso al crecimiento económico, tiene las ventajas adicionales de ser un canal de movilidad. Lamentablemente, tiempo después, una de las grandes asignaturas pendientes de la formación está en la posibilidad de obtener de forma objetiva la "tasa de retorno" de la formación. Pese a los sofisticados modelos matemáticos ensayados, éstos no han dejado de aproximarse tangencialmente al objetivo.

Una idea central en esta escuela es que los trabajadores acuden al mercado laboral con diferentes niveles de cualificaciones, que responden no sólo a las diferencias existentes entre sus capacidades innatas, sino también, y sobre todo, a que han dedicado diferentes cantidades de tiempo a adquirir esas cualificaciones, es decir, a invertir en capital humano.

Según Mato Díaz (1995: 2):

[...] la aportación más sustancial de esta perspectiva radica en la idea ya citada de entender la educación como una inversión. Los rendimientos de esa inversión vienen dados por las mejoras en la productividad, por lo que trabajadores con dotaciones diferentes de capital humano, representarían niveles de productividad, lo que supone abandonar la idea de que el trabajo, como factor productivo, es homogéneo.

No son muchos los economistas institucionalistas que han recogido este desafío y han tratado de hacerle frente, pero sí puede contarse con la obra de Piore (1973), esforzado en intentar abrir una línea teórica, donde, además de criticar a la escuela de la que procedía, cuestiona a sus antecesores por su desmesurado empirismo y su falta de sistematización teórica. Su trabajo teórico está íntimamente relacionado con la hipótesis del "mercado dual de trabajo» y con el estudio de la segmentación del mercado laboral. De ahí que muchas veces se piense que su teoría es la teoría de la segmentación, cuando, en realidad, su mejor encuadramiento sería el de una teoría institucionalista estructuralista. 
En última instancia, lo que está poniendo en cuestión es el supuesto conductual de la teoría del capital humano, según la cual el individuo se comporta «instrumentalmente», es decir, es capaz de distinguir entre medios y fines y de seleccionar los medios que satisfacen más eficazmente los fines, todo ello independientemente de la conducta de los demás individuos.

Otra de las críticas realizadas a esta escuela proviene de Blaug (1976: 827855), el cual parte del concepto del programa de investigación de Lakatos para tratar de responder a la pregunta de si el estatus empírico de la teoría del capital humano es el de un programa degenerante o progresivo. Sus conclusiones, sin llegar a ser totalmente negativas, no son excesivamente alentadoras para la teoría. Según Blaug, la adopción de la teoría del capital humano está relacionada con la concepción individualista del mundo de la teoría neoclásica, más que con criterios estrictamente teóricos, como apuntaba Piore.

\subsubsection{Escuela Bravermaniana}

Una segunda corriente de pensamiento, encabezada por Braverman, plantea cómo la implantación de la tecnología y la automatización de los procesos de trabajo producen la descualificación profesional a largo plazo.

Es la idea que desarrollará Braverman (1974) en su teoría del capital monopolista. Con ello, no debe caerse en el error de interpretarlo como alguien opuesto a los avances tecnológicos. Muy al contrario, ya que él mismo señala como siempre fue consciente de la marcha inexorable de los cambios tecnológicos, remarcando la idea de que la transformación del proceso de trabajo no sólo es inexorable, sino también necesaria para el progreso de la humanidad y su emancipación del hambre y otras formas de necesidad.

Esta es una de las conclusiones que se esconden tras el planteamiento teórico bravermaniano, en el que el capitalismo, tras un proceso de desarrollo histórico degenerativo, llega a un estadio que él denomina «capitalismo monopolista».

Es en esta fase donde el mercado se universaliza como consecuencia de su propia esencia: se expande a otros mercados orientándose deliberadamente hacia nuevas formas de dominación, a través del uso de todo tipo de bienes y servicios. Efectos no deseados, como un trabajo deshumanizado, degradado y copado por seres itinerantes faltos de motivación y entregados a su propia suerte y destino, son algunos de los aspectos negativos esta fase.

Es lo que, dicho en palabras de Finkel (1994: 254):

El nivel de destrezas y habilidades requeridos se reduce progresivamente bajo el capitalismo, porque los empleadores utilizan la descualificación de la fuerza de trabajo como un método de control de la misma.

De esta manera, Braverman indica (1974: 255):

Los trabajadores no sólo pierden control sobre los instrumentos de producción, sino que también pierden control sobre su propio trabajo y la manera en que lo desarrollan. 
Ahora bien, dentro de este análisis, deben considerarse las diferencias entre proletarización técnica e ideológica. Derber (1982) distingue entre estos dos conceptos y señala como la proletarización técnica implica pérdida de control sobre el conocimiento del proceso de trabajo y la proletarización ideológica alude a la pérdida de control sobre los objetivos y los propósitos del propio trabajo.

Es importante señalar que, tras esta lectura, se esconden una serie de principios más o menos razonables: disociar el proceso de trabajo de las habilidades de los trabajadores, separar la concepción y la ejecución del trabajo en el propio empleado $y$, por último, emplear el monopolio del conocimiento para controlar cada paso del proceso de trabajo y su forma de ejecución por parte del empleador.

Siguiendo este esquema, el trabajo administrativo pasa de ser un trabajo cualificado a degradarse, hasta que se convierte en un puesto descualificado, similar al de un operario de una empresa fabril, donde no hay opción a ejecutar el trabajo con una mínima capacidad de autonomía.

De todo este planteamiento, varias son las críticas que pueden reflejarse en él. No obstante, en honor a la verdad, dentro de su teoría hay un rasgo que, cuanto menos, atempera parte de sus afirmaciones y define a su modelo como no determinista, al entender que la tecnología por sí sola no produce descualificación, sino que es necesario que exista una ideología sobre la organización del trabajo que permita que los cambios tecnológicos generen efectos descualificadores. Según McDerment (1985: 66):

Es la política general de la empresa la que determinará las medidas a tomar y, sobre todo, la revalorización o la desvalorización de las cualificaciones como consecuencia de la introducción de nuevas tecnologías.

Con relación a las críticas directas, Wood (1989) adquiere una relevancia especial en este análisis, puesto que cuestiona por qué Braverman considera a Taylor como la panacea explicativa de la descualificación profesional, cuando Taylor no va más allá en su análisis, al centrarse en cómo puede reducirse la fatiga del trabajador durante el flujo laboral o cómo es posible mejorar el flujo de materiales en los procesos de fabricación.

Además, estos autores consideran la inexistencia empírica de experiencias concretas sobre este tema. A esto debemos añadir la consideración de que el hecho de que exista una estrategia gerencial (mental), no implica necesariamente que se haya implantado en la realidad.

Braverman asume que la respuesta de la clase obrera es homogénea a este proceso progresivo de descualificación, cuando esto no es rotundamente cierto. Pensemos por un momento en la composición de cualquier organización de producción de bienes y/o servicios, donde los perfiles profesionales son muy dispares, tanto como la recualificación o la descualificación, que sufre procesos paralelos.

Así pues, encontramos una posible conceptualización teórica del fenómeno de la descualificación profesional en los estudios pertenecientes al Brighton 
Labour Process Group, donde se especifican tres aspectos del proceso de descualificación:

a) La sustitución de los trabajadores cualificados por máquinas u operadores de maquinaria, la división y la subdivisión del trabajo, con lo cual se dejan ciertas tareas a unos pocos trabajadores cualificados y la fragmentación del resto a las tareas semicualificadas o descualificadas.

b) Este es, por tanto, un aspecto clave que limita parcialmente el contenido, el alcance y la dirección de la teoría bravermaniana y que deja sin contestar una cuestión importante: «iLa descualificación / recualificación / descualificación está asociada al puesto de trabajo o a la persona que lo ocupa?». No debe olvidarse que la descualificación profesional es la respuesta a un análisis multivariante, que puede ir paralelamente unida al propio trabajador de forma individual (nunca a un colectivo, al ser generalmente más heterogéneo de lo que parece), pero también hay casos en los que las combinaciones pueden ser divergentes, es decir, hay situaciones en las que un trabajador altamente cualificado ocupa un puesto que sobrepasa con creces o, viceversa, personas poco cualificadas que están llamadas a ocupar un puesto con un alto nivel de exigencia en cuanto a sus capacidades se refiere.

c) Por último, y como elemento de reflexión, es factible pensar que sí existe una capacidad por parte de los trabajadores para poder controlar sus condiciones en la empresa, y ello no implica que decidan sobre su futuro laboral. También es razonable intuir que la falta de capacidad y de control pueda ser determinante en su descualificación profesional.

\subsubsection{Escuela contingente}

Frente a estas dos grandes escuelas de pensamiento, cabe mencionar la denominada "teoría contingente». Uno de sus principales representantes, Spenner (1985), señala que la tecnología tiene un efecto mixto sobre las cualificaciones. En algunos casos, los estudios empíricos demuestran la existencia de tendencias descualificadoras, mientras que en otros es posible aducir que ciertos trabajos se recualifican profesionalmente.

Por ello, los impactos de las tecnologías en los niveles de cualificación no son simples, directos ni constantes en distintos ámbitos y empresas y, por tanto, no pueden considerarse aisladamente. Esto explica que la misma innovación en dos empresas diferentes puede alterar las cualificaciones de forma totalmente distinta.

Milkman y Pullman (1991) recogen la polarización de Spenner como resultado de un estudio realizado en los años ochenta en una fábrica de General Motors, en New Jersey, donde se indica como este fenómeno es más complejo de lo que aparentemente parece. Estos autores señalan como los factores organizacionales explican la polarización de las cualificaciones, más que la tecnología en sí misma.

Dentro de esta tradición contingentalista, Penn y Scattergood (1978) mantienen su teoría compensatoria de las cualificaciones, en la que se definen cinco 
cuestiones clave: en primer lugar, la teoría sugiere que el cambio tecnológico genera al mismo tiempo cualificación y descualificación; en segundo lugar, en las sociedades capitalistas desarrolladas, estos efectos se amplifican a escala internacional; en tercer lugar, los cambios tecnológicos tienden, por un lado, a la descualificación en las tareas directas de producción, pero, al mismo tiempo, desarrollan y estimulan las tareas auxiliares asalariadas con la instalación, el mantenimiento y la programación de la maquinaria automatizada; en cuarto lugar, este planteamiento postula que el cambio tecnológico tiende a favorecer a ciertos grupos, mientras que perjudica a otros, y, por último, este modelo advierte que el cambio técnico afecta a las formas tradicionales de la división del trabajo y por ello comporta simultáneamente amenazas y oportunidades para las organizaciones de representación sindical.

Por último, Boyer (1986b) y, en general, toda la escuela regulacionista aprecian como «el Régimen de Acumulación de la Especialización (o Automatización) Flexible», caracterizado por: unidades de producción más pequeñas y menos especializadas, escaso control de la jerarquía y los procesos de participación de los trabajadores en el proceso de trabajo, etc., no implican que el nivel de empleo dependa exclusivamente de la introducción de las nuevas tecnologías. Dicho de otra forma, la tecnología no hace sino provocar la afloración de las carencias formativas de los individuos que, de otra forma, no habrían sido detectadas.

\section{Las competencias}

Si hasta este momento se ha tenido la oportunidad de realizar una aproximación al concepto de cualificación profesional, a través de diferentes acepciones del término y el tratamiento que diferentes escuelas le han dado, es momento de intentar contextualizar el significado del constructo competencia e interpretar terminológicamente su contenido desde una triple dimensión: la conceptual, la académico-docente y la que se relaciona con el mercado laboral.

\subsection{Aproximación inicial al término competencia}

McClelland (1973) define inicialmente el término competencia como: «aquello que realmente causa un rendimiento superior en el trabajo». Dicho autor antepone su significado y su dimensión al enfoque educativo para ponerlo en correspondencia con otros elementos alternativos, como el género, la etnia o la clase social, a fin de medir el rendimiento laboral de la persona en el contexto organizativo.

Desde entonces, muchos han sido los desarrollos posteriores que se han realizado tomando como referencia esta primera definición. Casi de foma coincidente en el tiempo, Bloom (1975) hace referencia a las competencias, aunque desde la óptica educativa, al mencionar que la «enseñanza basada en competencias» se asienta en cinco grandes postulados: 
- Todo aprendizaje es esencialmente individual.

- El individuo, al igual que cualquier sistema, se orienta hacia la consecución de un conjunto de metas a lograr.

- El proceso de aprendizaje es más fácil cuando el individuo sabe qué es exactamente lo que se espera de él (algo no tan sencillo como pudiera parecer)

- El conocimiento preciso de los resultados contribuye a facilitar el proceso de aprendizaje.

- Es más probable que un alumno haga lo que se espera de él y lo que él mismo desea, si tiene la responsabilidad en las tareas de aprendizaje.

Los principios enumerados por Bloom se convierten, tiempo después, en la referencia a partir de la cual se construyeron los modelos de educación y formación basados en competencias, tanto en Estados Unidos como en Gran Bretaña. Fue precisamente en este último país donde el modelo fue aceptado y se consolidó hasta hoy en día. La competencia era entendida como el resultado necesario de la formación (Tuxworth, 1989).

\subsection{Interpretaciones terminológicas}

Gracias a los originales puntos de partida que tanto McClelland como Bloom han presentado, este es el momento en el que resulta necesario observar las diferentes evoluciones del concepto competencia. Es interesante hacerlo desde tres puntos de vista no necesariamente contrapuestos, sino, todo lo contrario, complementarios.

En primer lugar, se hablará desde un punto de vista conceptual, matizando la rica complejidad de aproximaciones al concepto. En segundo término, se examinará la variedad polisémica que ofrece el término competencia desde un punto de vista académico-docente y, por último, se abordará la cuestión desde el prisma del mercado laboral.

Desde este esquema, parece lógico pensar que los diferentes escenarios planteados deben guardar cierto equilibrio interno y cómo uno con otro terminan de completar un modelo explicativo más complejo y plural.

\subsubsection{Desde un punto de vista conceptual}

Según Boyatzis (1982), el término competencia se define como: «Característica subyacente de la persona, que esta causalmente relacionada con un criterio de referencia de actuación exitosa en el puesto de trabajo o en otra situación». Lo que, al menos, puede hacer reparar en tres aspectos clave:

- Caracteristica subyacente significa que forma parte de la personalidad y puede predecir una amplia variedad de comportamientos, tanto en el trabajo como en la vida personal.

- Causalmente relacionada, porque es la causa de algo o predice un comportamiento. 
- Criterio de referencia de actuación exitosa explica que las competencias podrán predecir cómo se realizará una actuación (bien, mal, regular; mejor, peor) a partir de unos criterios estandarizados.

Estos aspectos hacen atisbar una diferenciación de las competencias en dos grandes grupos:

- Competencias diferenciadoras: caracterizadas por distinguir un desempeño superior de un desempeño medio o estándar.

- Competencias umbral o esenciales: relacionadas con el logro de una actuación media o mínimamente adecuada.

De este modo, las competencias pueden consistir en:

- Motivos, entendidos como la necesidad subyacente o una forma de pensar que impulsa, orienta y selecciona la conducta de una persona. Por ejemplo: la necesidad de un logro.

- Rasgos de carácter o predisposición general a conducirse o a reaccionar de un modo determinado. Por ejemplo: confianza en uno mismo, autocontrol, resistencia al estrés o a la frustración.

- Concepto de uno mismo o lo que uno piensa, lo que valora y/o lo que está interesado a realizar, orienta el patrón de comportamiento individual.

- Conocimientos o lo que se sabe sobre una técnica, ciencia y/o habilidad, determina la capacidad de actuación y, por tanto, condiciona al comportamiento.

- Capacidades cognitivas y de conducta, ya sean ocultas (razonamiento deductivo) u observables (escucha activa).

En suma, un conjunto de matizaciones orientadas a una mejor definición del término competencia que, tiempo más tarde, no pasan inadvertidas a Lawler (1994), a quien se debe su aplicabilidad práctica al mundo empresarial, o como las ventajas de pasar a una empresa gestionada por competencias implica el paso de la burocratización a un modelo sistémico-organizativo.

En nuestro país, los trabajos realizados por Pereda y Berrocal (2001), inspirados en las ideas de Le Boterf, Barzucchetti y Vincent (1993), describen cinco elementos que, en su opinión, ayudan a definir la naturaleza de la competencia.

Estos elementos se corresponderían con cinco tipos de saberes:

- Saber, o conocimientos que posee la persona y que le permitirán llevar a cabo los comportamientos incluidos en la competencia.

- Saber hacer, o la capacidad que tiene esa persona para aplicar aquellos conocimientos orientados a la solución de problemas o conflictos.

- Saber estar, o la realización de esos comportamientos en función de los procedimientos propios de la organización. 
- Querer hacer o querer llevar a cabo los comportamientos que articulan a la competencia, lo que alude directamente a la motivación del individuo.

- Poder hacer o las características de la organización que permiten al individuo disponer de los medios y de los recursos pertinentes necesarios para desarrollar su competencia.

Ya más recientes en el tiempo son los estudios de naturaleza epistemo-metodológica de De Haro (2004), en un intento por clasificar la infinidad de modelos -en opinión de éste, tantos como autores- que se han ido sucediendo en el transcurso del tiempo. En este sentido, la propuesta que realiza se basa en la comprensión del término competencia, según sea entendida como variable dependiente o independiente.

En este segundo caso, al ser caracterizada como variable independiente, la competencia es contemplada como causa u origen del desempeño o del resultado. A su vez, las causas que determinan el desempeño eficaz y eficiente de los trabajadores es de varios tipos: el que se basa en rasgos, en conductas o en una combinación de ambos. De este modo, concluye señalando como la competencia es un constructo con el que se califican comportamientos relacionados entre sí, y éstos son los responsables directos de un resultado excelente en el desempeño del puesto de trabajo.

\subsubsection{Desde un punto de vista académico-docente}

No quedaría completo nuestro análisis si, desde el punto de vista competencial, no hubiera una mínima reflexión en dos direcciones: desde la perspectiva del profesorado y desde la del alumnado.

\subsubsection{El profesorado}

Debemos remitirnos al anexo I del RD 1312/2007, donde el conjunto de méritos que deben ser valorados se materializa en más de cincuenta elementos que deben ser analizados por las comisiones de acreditación de la ANECA y que, por tanto, deben ser aportados por los candidatos. En este sentido, se establece una valoración máxima para las dimensiones y unos umbrales, así como una valoración de puntos mínimos por sexenios que determina un porcentaje muy elevado de las solicitudes a la acreditación para concursos al cuerpo de catedráticos de universidad. Sería prolijo entrar en su análisis, pero no tanto recoger algunos de los principales resultados obtenidos en la acreditación para el cuerpo de catedráticos de universidad (cuadro 1) y profesores titulares (cuadro 2) y hacerse eco, aunque fuera mínimamente, de las conclusiones de este trabajo, donde se propone hacer una reflexión profunda de los aspectos considerados $\mathrm{y}$, mediante la correspondiente metaevaluación, proponer un conjunto de mejoras que muy probablemene requerirán la modificación del $\mathrm{RD}$ 1312/2007 a medio plazo. En suma, un debate abierto acerca del sistema competencial y su mejora continua, en un marco donde el alumnado se convierte en la piedra angular del sistema 


\subsubsection{El alumnado}

Tiempo más tarde que en otros países de nuestro entorno, en 2002, nuestro país establece el Sistema Nacional de Cualificaciones y Formación Profesional, con lo cual el Catálogo Nacional de Cualificaciones Profesionales se convierte en el eje fundamental del sistema. Como resultado de la relevancia estratégica que adquiere la denominación de competencia, ésta se define como: «el conjunto de conocimientos y capacidades que permiten el ejercicio de la actividad profesional conforme a las exigencias de la producción y del empleo» (LO 5/2002).

Algo después, y con motivo de la entrada en vigor del RD 1128/2003, por el que se regula el Catálogo Nacional de Cualificaciones Profesionales, se define a la cualificación profesional (con otros matices complementarios a los anteriores) como: «el conjunto de competencias profesionales con significación para el empleo que pueden ser adquiridas mediante formación modular u otros tipos de formación, así como a través de la experiencia laboral». Es la ANECA quien, haciéndose eco del sistema competencial, toma

Cuadro 1. ANECA. Solicitudes resueltas para cuerpo de catedrático de universidad

\begin{tabular}{|l|c|c|c|c|c|}
\hline Comisión CU & Total & Positivos & $\%$ Pos. & Negativos & $\%$ Neg \\
\hline Artes y humanidades & 473 & 346 & 73,15 & 127 & 26,85 \\
\hline Ciencias & 703 & 623 & 88,62 & 80 & 11,38 \\
\hline Ciencias de la salud & 335 & 252 & 75,22 & 83 & 24,78 \\
\hline Ciencias sociales y jurídicas & 523 & 358 & 68,45 & 165 & 31,55 \\
\hline Ingeniería y arquitectura & 344 & 264 & 76,74 & 80 & 23,26 \\
\hline Total & 2.378 & 1.843 & 77,50 & 535 & 22,50 \\
\hline
\end{tabular}

Fuente: datos a 31 de mayo de 2009. Elaboración propia a partir del informe del Patronato sobre la acreditación nacional para el acceso a los cuerpos de profesorado universitario.

Cuadro 2. ANECA. Solicitudes resueltas para profesor titular de universidad

\begin{tabular}{|l|c|c|c|c|c|}
\hline Comisión PTU & Total & Positivos & \% Pos. & Negativos & $\%$ Neg \\
\hline Artes y humanidades & 389 & 314 & 80,72 & 75 & 19,28 \\
\hline Ciencias & 619 & 505 & 81,51 & 114 & 18,42 \\
\hline Ciencias de la salud & 408 & 273 & 66,91 & 135 & 33,09 \\
\hline Ciencias sociales y jurídicas & 511 & 317 & 62,04 & 194 & 37,96 \\
\hline Ingeniería y arquitectura & 496 & 390 & 78,63 & 106 & 21,37 \\
\hline Total & 2.423 & 1.799 & 74,25 & $\mathbf{6 2 4}$ & 25,75 \\
\hline
\end{tabular}

Fuente: datos a 31 de mayo de 2009. Elaboración propia a partir del informe del Patronato sobre la acreditación nacional para el acceso a los cuerpos de profesorado universitario. 

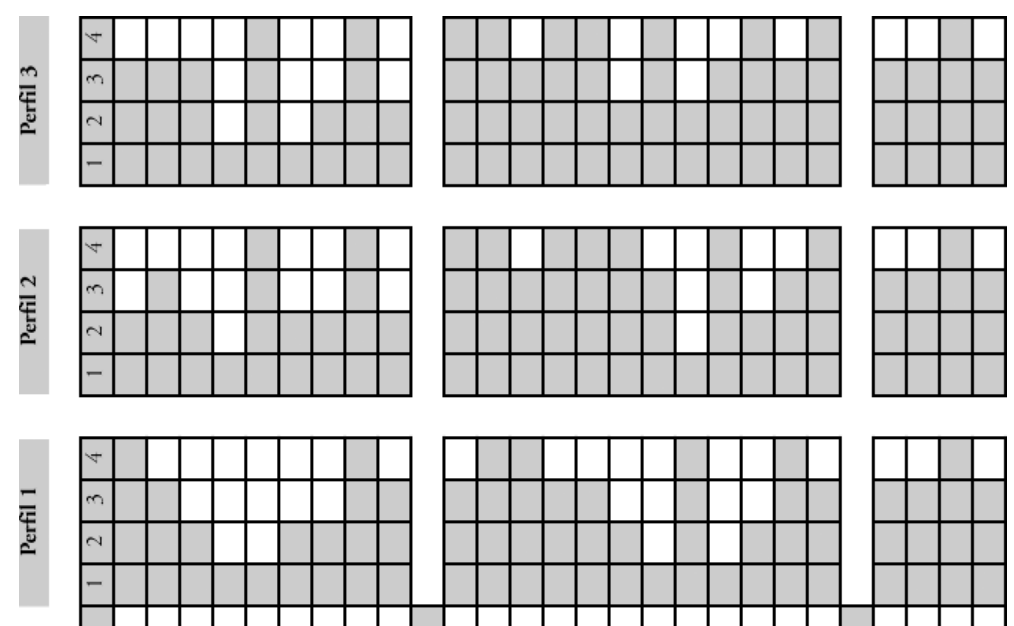

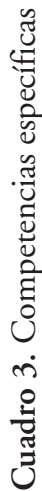

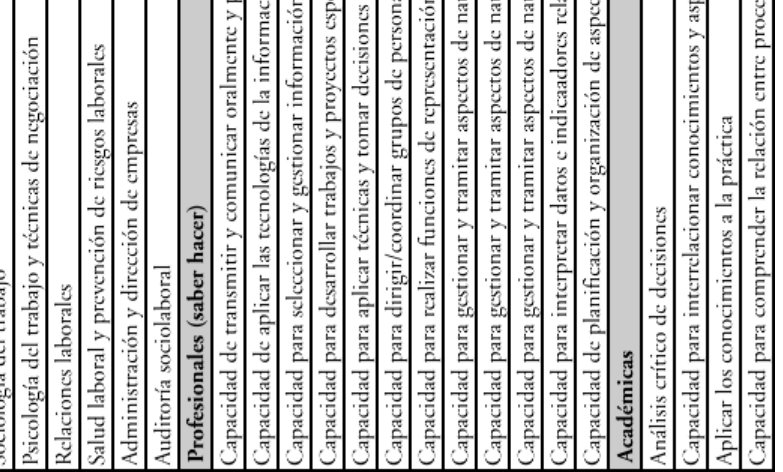

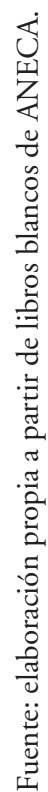



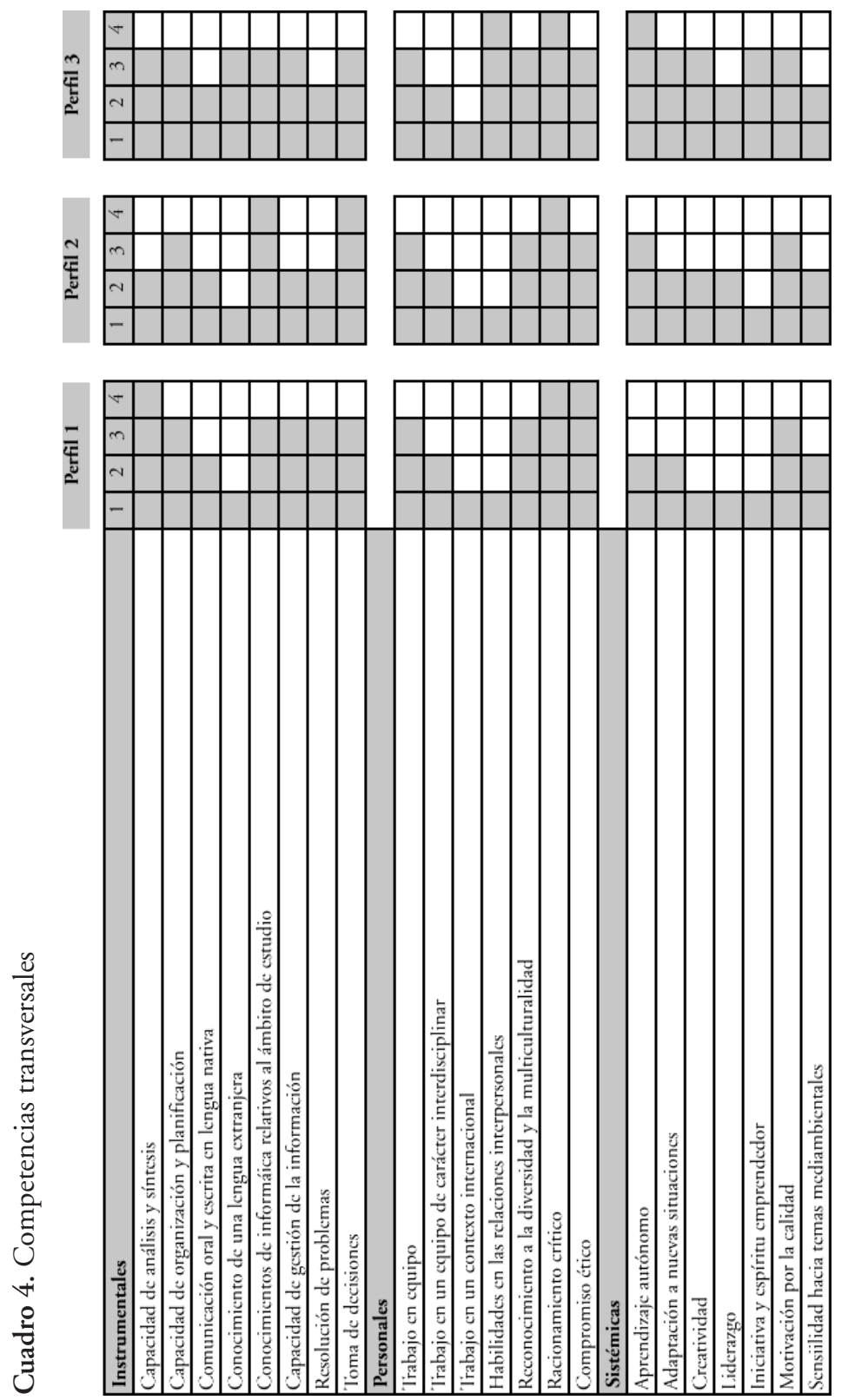

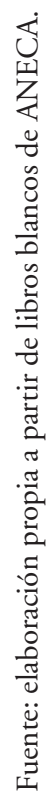


como referencia su espíritu, para modelizar un conjunto de competencias que den sentido a los nuevos títulos de grado. Esta nueva filosofía, inspiradora del actual sistema educativo de formación superior, toma como referente los llamados «libros blancos», que se construyen en orden a una tipología competencial:

- Las competencias especificas: relacionadas directamente con el contenido de los grados que representan a las nuevas titulaciones del Plan Bolonia. Estas competencias sirven de referencia para caracterizar a los perfiles profesionales. Básicamente, recogen los siguientes aspectos: conocimientos disciplinares (saber), competencias profesionales (saber hacer) y competencias académicas. En el cuadro adjunto (cuadro 3), pueden apreciarse algunas de estas competencias especificas para el estudio de un hipotético perfil del área de Administración con diferentes especializaciones.

- Las competencias transversales, caracterizadas a través de tres dimensiones (instrumentales, personales y sistémicas), son aquellas que complementan a las denominadas «específicas» (ver cuadro 4). Su propia naturaleza las hace que sean más comunes a más de un perfil.

Son, por tanto, un conjunto de dimensiones que, oportunamente puestas en correspondencia con el perfil académico que la persona precisa, servirán para el desarrollo académico del individuo.

\subsubsection{Desde un punto de vista del mercado laboral}

$\mathrm{Si}$ hasta este momento se ha comentado, aunque someramente, el estudio competencial desde una perspectiva conceptual y académica, es oportuno abordar otra dimensión: la relativa al mercado laboral.

Parece evidente que una definición competencial sin una conexión real con el mercado laboral se quedaría en un conjunto de buenas intenciones, pero no en una realidad social. Es por ello que el análisis de competencias desde la perspectiva laboral se hace necesaria, aunque sea de un modo aproximativo.

En este contexto, la oferta y la demanda de la fuerza de trabajo obligan a llegar a un punto de encuentro donde conciliar posiciones. En este sentido, y según el informe ejecutivo realizado por la Unidad de Estudios de la ANECA, denominado El profesional flexible en la sociedad del conocimiento (2007a), menciona cinco grandes familias de competencias requeridas por el puesto de trabajo y, por extensión, en el mercado laboral. Son competencias relacionadas con el conocimiento, el análisis y la innovación, la gestión del tiempo, la organización y la comunicación.

Estas cinco familias son las que, en su máximo desarrollo, dan lugar a diecinueve competencias (ver cuadro 5) que son prácticamente coincidentes, tanto desde la exigencia del mercado laboral como desde la perspectiva del empleador. 
Cuadro 5. Competencias requeridas por el mercado laboral y el empleador

\begin{tabular}{|l|l|}
\hline \multicolumn{2}{|c|}{ Competencias } \\
\hline Dominio de su área o disciplina. & Capacidad para trabajar en equipo. \\
\hline $\begin{array}{l}\text { Conocimientos de otras áreas o } \\
\text { disciplinas. }\end{array}$ & $\begin{array}{l}\text { Capacidad para movilizar las } \\
\text { capacidades de otros. }\end{array}$ \\
\hline Pensamiento analítico. & Capacidad para hacerse entender. \\
\hline $\begin{array}{l}\text { Capacidad para adquirir con rapidez } \\
\text { nuevos conocimientos. }\end{array}$ & $\begin{array}{l}\text { Capacidad para hacer valer tu } \\
\text { autoridad. }\end{array}$ \\
\hline $\begin{array}{l}\text { Capacidad para negociar de forma eficaz. } \\
\text { Capacidad para utilizar herramientas } \\
\text { informáticas. }\end{array}$ \\
\hline \begin{tabular}{l} 
Capacidad para rendir bajo presión. \\
Capacidad para encontrar nuevas \\
ideas y soluciones. \\
\hline oportunidades.
\end{tabular} & $\begin{array}{l}\text { Predisposición para cuestionar ideas } \\
\text { propias o ajenas. }\end{array}$ \\
\hline $\begin{array}{l}\text { Capacidad para coordinar actividades. } \\
\text { en idiomas extranjeros. }\end{array}$ & $\begin{array}{l}\text { Capacidad para presentar en público } \\
\text { productos, ideas o informes. }\end{array}$ \\
\hline Capacidad para usar el tiempo de forma & $\begin{array}{l}\text { Capacidad para redactar informes } \\
\text { o documentos. }\end{array}$ \\
\hline
\end{tabular}

Fuente: ANECA-REFLEX (2007a). El profesional flexible en la Sociedad del Conocimiento (informe ejecutivo) y ANECA-REFLEX (2007b). Informe empleadores (titulados universitarios y mercado laboral).

\section{Competencias y cualificacion}

A la vista de lo anterior, cabe preguntarse si, finalmente, puede establecerse una relación entre el concepto de competencia y el de cualificación. Aunque intuitivamente pueda resolverse este aparente conflicto, no está de más revisar algunos de los comentarios que han realizado diferentes autores, sobre todo en la década de 1990.

Alex (1991) resuelve esta conexión señalando que si la cualificación responde a una dimensión personal, la competencia forma parte de ella (de la cualificación) y responde a una dimensión social. En esta misma línea, Alaluf y Stroobants (1994) señalan como la competencia forma parte de la cualificación 
Cuadro 6. Cualificaciones y competencias

\begin{tabular}{|l|l|}
\hline \multicolumn{1}{|c|}{ Cualificaciones } & \multicolumn{1}{c|}{ Competencias } \\
\hline $\begin{array}{l}\text { Capacidades adquiridas y reconocidas } \\
\text { por el sistema educativo. }\end{array}$ & $\begin{array}{l}\text { Capacidades adquiridas por } \\
\text { la experiencia vitl (vida personal } \\
\text { y profesional). }\end{array}$ \\
\hline $\begin{array}{l}\text { Cenrado en el conocimiento de materias } \\
\text { y/o disciplinas. }\end{array}$ & $\begin{array}{l}\text { Centrado en la producción de } \\
\text { resultados, conectado en mayor o } \\
\text { menor medida a un contexto } \\
\text { personal y/o profesional concreto. }\end{array}$ \\
\hline $\begin{array}{l}\text { Centros educativos preferentemente } \\
\text { identificables. }\end{array}$ & $\begin{array}{l}\text { El lugar de formación son a menudo } \\
\text { las circunstancias vitales. }\end{array}$ \\
\hline $\begin{array}{l}\text { La duración del aprendizaje está } \\
\text { predifinida. }\end{array}$ & $\begin{array}{l}\text { El aprendizaje es independiente de su } \\
\text { duración. }\end{array}$ \\
\hline $\begin{array}{l}\text { Presenta una dimensión colectiva para } \\
\text { las clasificaciones profesionales. }\end{array}$ & $\begin{array}{l}\text { Presenta una dimensión individual que, } \\
\text { por ejemplo, no es representativa en } \\
\text { figuras como el convenio colectivo. }\end{array}$ \\
\hline
\end{tabular}

Fuente: adaptación de «Cualificaciones y competencias» (Colardyn, 1996: 54).

y como sirve para demostrarla o ponerla a prueba. En este sentido, son impagables las reflexiones que Stroobants (1991) realiza cuando profundiza en la innegable construcción social de la cualificación.

Estos aspectos nos llevan a reflexionar acerca de la naturaleza estática o dinámica de ambos conceptos. De este modo, autores como Gallart y Jacinto (1996) y, tiempo después, Stroobants (1999) coinciden en señalar un carácter más estable o estático para la cualificación y más variable o dinámico para la competencia.

Colardyn (1996) profundiza en matices especialmente singulares que le llevan a plantear el escenario de la cualificación con lo formal, mientras que el competencial lo relaciona con lo no «formal» o, si se prefiere, con lo «informal» (ver cuadro 6).

Le Boterf (2000), en una visión más estratégica, aborda el necesario maridaje entre competencias y cualificación, ya que facilitaría la desigualdad social y la posibilidad de contribuir de un modo cierto a la empleabilidad del sistema.

En este sentido, la empleabilidad del sistema no deja de ser una cuestión armónica, donde la adecuación entre personas y puestos de trabajo no puede entenderse sin un necesario equilibrio entre cualificación y desarrollo competencial. En la medida en que exista una correspondencia entre estas variables, será factible la construcción de una sociedad más igualitaria y abierta al vértigo del cambio. 


\section{Hacia una definición del término competencia}

Es oportuno aclarar que nuestra posición inicial no pretende en modo alguno cuestionar ninguna de las anteriores construcciones del significado de competencia. Más bien al contrario, se tratará de potenciar las diferentes y siempre interesantes interpretaciones realizadas, lo cual favorecerá el debate terminológico del constructo.

Tras una primera aproximación inicial al término competencia a través de la delgada y sinuosa línea que demarca los conocimientos, las capacidades y las habilidades, es nuestra intención remarcar el peso de lo individual frente al de lo grupal o, si se quiere, lo "psico» frente a lo «socio», en un intento modelizador del constructo que se presenta.

\subsection{Conocimientos, capacidades y habilidades}

Desde un posicionamiento inicial, tres son —entendemos — las dimensiones que pueden ayudar a modelizar el término competencia, con independencia del ámbito (académico y/o profesional) que se esté considerando (ver la figura 1).

La primera dimensión guarda relación con los conocimientos (reglados o no reglados) con los que una persona cuenta desde una perspectiva teórico-práctica. Estos conocimientos, como se ha indicado, pueden estar legitimados bien desde la formación reglada o desde los aspectos informales que se recogen desde las vivencias y las experiencias que jalonan la vida cotidiana. El componente

Figura 1. Representación competencial

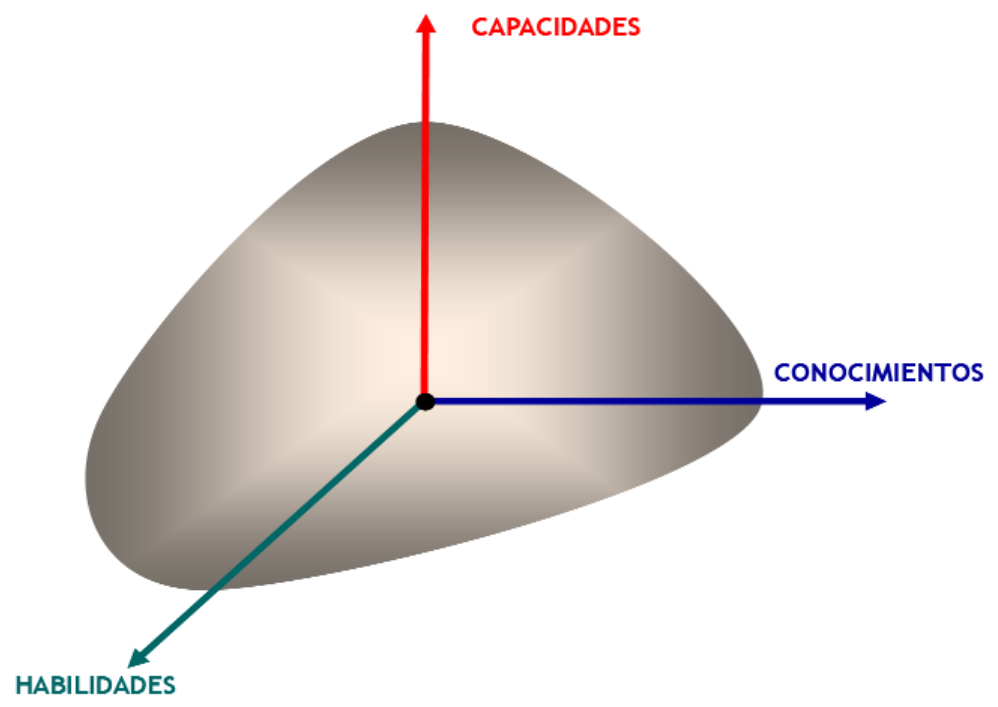

Fuente: elaboración propia. 
teórico (lo que es o debe ser) junto al práctico (lo que hago o lo que se debe hacer) es una aproximación inicial a esta dimensión.

Existe una segunda dimensión vinculada a las capacidades, entendidas como el potencial relacionado con las características naturales del individuo para gestionar determinadas situaciones. Es importante precisar que, en principio, si bien todo el mundo nace con éstas, ello no comporta un desarrollo uniforme en el mismo rango de individuos, lo que apunta a la influencia de aspectos ecológicos y ambientales para explicar las diferencias existentes en su desarrollo.

Posibles ejemplos de esta dimensión pueden ser: la motivación por el logro, la preocupación por el orden y la claridad, la iniciativa y la proactividad, la construcción de relaciones, el pensamiento conceptual y analítico, el autocontrol personal y la confianza en uno mismo, por citar sólo algunos.

La tercera dimensión, en algunos casos colindante con la anterior, hace que la frontera que las separa sea muy tenue, razón por la cual algunas habilidades pueden verse a menudo solapadas con las capacidades. Aun con todo, el hablar de habilidades comporta referirse a ciertas destrezas no necesariamente innatas, ni tan siquiera sospechadas por el propio individuo, que, al ser «descubiertas» $y$ entrenadas (posiblemente por lo específico de su contenido, alcance y dimensión), permiten un mejor desarrollo a la persona en su relación con el entorno. En esta dirección, podría hablarse de los siguientes aspectos: presentación de ideas, trabajo en equipo, dirección de reuniones, resolución de conflictos y capacidad negociadora, organización y planificación de actividades, entre otras.

De admitirse este sencillo esquema, la posibilidad de contemplar los conocimientos, las capacidades y las habilidades como marco referencial sobre el que diseñar, desarrollar e implantar un modelo de gestión por competencias parece más al alcance de la mano.

\subsection{Individualidad y grupalidad}

Tras esbozar incialmente el germen de lo que puede aventurarse como una competencia, surge un segundo escenario de análisis en el que detenerse. Las competencias, con independencia de su significado inicial, presentan una naturaleza diferente según el carácter individual o grupal en el que se enmarcan.

En otras palabras, la esencia de las competencias guarda una estrecha relación con el lugar en el que se gestan y en el que se desarrollan, psicológicamente o sociológicamente (ver cuadro 7)

Resultado de esta diferenciación, debería hablarse, por tanto, de dos grandes tipologías. En relación con las caracterizadas por el carácter individual o psicológico, se mencionan las siguientes:

- Iniciativa y proactividad: predisposición a crear las condiciones que, si bien no aseguran el desarrollo de los acontecimientos, procuran disminuir o atenuar las circunstancias de naturaleza adversa a lo inicialmente proyectado.

- Adaptación y flexibilidad: facultad por la que la persona es capaz de amoldarse al entorno, a la situación y/o a las circunstancias, sin que ello genere 
Cuadro 7. Escenarios competenciales

\begin{tabular}{|l|l|}
\hline \multicolumn{1}{|c|}{ Individuales } & \multicolumn{1}{c|}{ Grupales } \\
\hline Iniciativa y proactividad & Comunicación efectiva \\
\hline Adaptación y flexibilidad & Trabajo en equipo \\
\hline Motivación & Negociación \\
\hline Análisis de problemas y toma de decisiones & Liderazgo \\
\hline
\end{tabular}

Fuente: elaboración propia.

contradicciones o conflictos entre sí mismo, el puesto que ocupa y el medio en el que debe desenvolverse.

- Motivación: impulso y/o ánimo necesario para conseguir los fines personales propuestos y, por extensión, los del equipo de trabajo.

- Análisis de problemas y toma de decisiones: posición relacionada con la evaluación de situaciones, enmarcadas en un contexto de litigio, donde se hace necesaria la solución del conflicto en tiempo y forma.

Por otro lado, las caracterizadas por el carácter grupal o sociológico son las siguientes:

- Comunicación efectiva: representación que permite enviar, recibir y procesar datos e informaciones en un contexto interindividual, lo cual favorece la adquisición, la estimulación y el desarrollo de los comportamientos grupales orientados hacia un fin determinado.

- Trabajo en equipo: desarrollo de las facultades interindividuales que permiten la consecución de resultados en orden a los objetivos propuestos por la organización, lo cual optimiza los medios humanos y técnicos puestos a su alcance.

- Negociación: conjunto de técnicas orientadas a la obtención del máximo provecho de las personas representadas en situaciones de confrontación, donde, a fin de buscar una contraprestación satisfactoria para las partes, generalmente debe cederse en las posiciones.

- Liderazgo: facultad basada en persuadir, orientar e influir emocional y técnicamente a los individuos que forman parte del grupo, a fin de buscar su desarrollo personal y profesional.

\subsection{La integración de escenarios y definición del término}

Sobre la base de los supuestos anteriores, en los que los conocimientos, las capacidades y las habilidades permiten caracterizar el término competencia 
junto al carácter individual o grupal, es el momento de ver sus posibles interconexiones.

Desde una perspectiva holística, la concepción de cada realidad es un todo distinto a la suma de las partes. En este sentido, la construcción del término competencia es algo más que la suma de escenarios que concurren en el estudio del modelo explicativo.

En otras palabras, la definición del término competencia en ese marco de referencia bidimensional que categoriza, por un lado, a los conocimientos, las capacidades y las habilidades y, por otro, a la naturaleza de lo individual y lo grupal, no hacen sino generar un efecto sinérgico, en el que el conjunto total de lo obtenido es algo más que la suma aislada de las partes.

Puestos a enunciar el término competencia, este guarda relación con la adquisición, la estimulación y el desarrollo de un conjunto de conocimientos, capacidades y habilidades que, tanto desde una perspectiva individual como grupal, favorecen la cualificación personal y profesional de la persona.

Ahora bien, si esta puede ser una definición del término competencia, ¿cuál es la conexión con el de cualificación? Resulta evidente que, según la escuela de pensamiento, la caracterización del término cualificación gira en torno a diferentes planteamientos.

Desde nuestra percepción particular, entendemos por cualificación el nivel competencial que registra un individuo con referencia a las necesidades que le exige el entorno donde este interactúa. En otras palabras, la cualificación es una variable que depende de la competencia.

En la medida en que el individuo adquiera un mayor rango competencial, mayores serán sus posibilidades de adecuarse al entorno o, si se prefiere, al ecosistema y, más concretamente, a los requerimientos que de él se exigen.

La cuestión clave, sin embargo, gravita ante las posibles inadecuaciones entre la cualificación exigida y el nivel competencial existente. Cuando la cualificación requerida supera las competencias que aporta la persona (o cuando las competencias quedan por debajo del umbral de la cualificación), podría hablarse de infracualificación, mientras que cuando la cualificación solicitada queda por debajo de las competencias (o cuando las competencias se sitúan por encima de la cualificación), se hablaría de sobrecualificación en el puesto de trabajo.

En suma, un conjunto de desequilibrios en esa adecuación teórica entre persona y puesto de trabajo, con el trasfondo de la cualificación y las competencias profesionales.

\section{Conclusiones}

No siempre es tarea sencilla redefinir conceptos de todos conocidos pero que comportan una importante carga ideológica. A este análisis, no escapa ni muchísimo menos la cualificación profesional que se manifiesta a través de la actuación cotidiana del individuo en el puesto de trabajo. Prueba de ello son las innumerables interpretaciones de diferentes escuelas de pensamiento que, desde 
los teóricos del capital humano hasta Braverman, pasando por la Postura Contingente, exploran diferentes respuestas al escenario donde se dirime la cuestión.

Es en estas ocasiones cuando la definición de un término precisa del auxilio de otro, en este caso - y siempre desde nuestra perspectiva particular-, la cualificación no podría entenderse sin el referente del término competencia.

La construcción del término competencia es algo más que la suma de escenarios que concurren en el estudio del modelo explicativo. Es decir, hablar de competencia es acudir inicialmente — sólo inicialmente- a un espacio bidimensional que categoriza, en un eje, a los conocimientos, las capacidades y las habilidades y, en el otro, a lo individual y grupal, lo cual genera un efecto multiplicador, en el que, a la postre, el conjunto total de lo obtenido es algo más que la suma aislada de sus partes.

Desde nuestra comprensión particular, la cualificación — sin negar su construcción social - depende o está influida por el nivel competencial que registra un individuo con referencia a las necesidades que le exige el entorno donde este se encuentra. Es en este entorno donde otras variables donde el género, la edad, la etnia y tantas otras ampliarían el ámbito de este análisis, sin que sea el objetivo prioritario de este trabajo.

En un intento por definir la cualificación, ésta puede entenderse como el nivel competencial que registra un individuo con referencia a las necesidades que le exige el entorno donde este interactúa. En otras palabras, la cualificación es una variable dependiente de la competencia.

Por ello, en la medida en que el individuo adquiera un mayor rango competencial, mejores serán sus posibilidades de supervivencia.

Cuestión aparte son los posibles desequilibrios que pueden producirse cuando la cualificación requerida supera o no alcanza a las competencias que presenta la persona, lo cual da lugar a la infracualificación o sobrecualificación, respectivamente, de la persona en el puesto de trabajo.

Se trata de un fenómeno de polarización formativo, sometido a las variables leyes de la incertidumbre, donde no siempre las condiciones de partida de los diferentes colectivos son homogéneas y donde el darwinismo social puede ser clave en la explicación del fenómeno.

\section{Bibliografía}

Alaluf, M. (1988). Crise et representations du travail: la classe du compromis? Congreso Internacional sobre la Teoría de la Regulación (comunicación). Barcelona.

Alaluf, M. y STROobants, M. (1994). «iMoviliza la competencia al obrero?». Revista Europea de Formación Profesional, 1, 46-55.

AlEX, L. (1991). «Descripción y registro de las cualificaciones. El concepto de cualificación». Formación Profesional, 2, 23-27.

Alonso, L.; FernÁNDEZ, C. y NySSEN, J. (2009). El debate de las competencias. Madrid: ANECA.

ANECA (2009). Informe al Patronato sobre la acreditación nacional para el acceso a los cuerpos de profesorado universitario. Madrid. 
ANECA / REFLEX (2007a). El profesional flexible en la sociedad del conocimiento (informe ejecutivo). Madrid.

- (2007b). Informe Empleadores (Titulados Universitarios y Mercado Laboral). Madrid.

BARCET, A.; LE BAS, C. y MERCIER, C. (1985). Le savoir faire et changementes techniques. Lyon: Presses Universitaires de Lyon.

BECKER, G. (1964). Human Capital: a theoretical and empirical analysis with special reference to education. Nueva York: Columbia University Press.

Bessant, J. (1992). Microelectrónica y cambio en el trabajo: Experiencias en la aplicación de la microelectrónica y cambios en el trabajo, el comercio, las oficinas y los servicios de salud. Madrid: Ministerio de Trabajo.

Blaug, M. (1976). "The empirical status of human capital theory: A slightly jaundiced survey». Journal of Economic Literature, XIV (3), 827-855.

Bloom, S. (1975). Evaluación del aprendizaje. Buenos Aires: Troquel.

Boyatzis, R. (1982). The competence manager. Nueva York: John Wiley \& Sons.

BOYER, R. (1986a). «Nuevas tecnologías y empleo en los 80». En: OMINAMI, C. (comp.). El sistema internacional y América Latina. La tercera revolución industrial. Impactos internacionales del actual viraje tecnológico. Buenos Aires: Grupo Editor Latinoamericano.

- (1986b). La flexibilidad del trabajo en Europa. Madrid: Ministerio de Trabajo.

Bowman, M. (1963). «Educational Shortage and Excess». Canadian Journal of Economic and Political Science, 29, 446-61.

Braverman, H. (1974). Trabajo y capital monopolista: la degradación del trabajo en el siglo XX. México: Editorial Nuestro Tiempo.

CAjA, A. (2010). «La formación de los directivos tecnológicos». En: BLANCO, J. (coord.). Formación de directivos. Madrid: Pearson Educación.

CASTILlo, J. (1987). «Hacia un diseño conjunto de las transformaciones productivas: condiciones de trabajo y nuevas tecnologías». En: GARMENDIA, J.; NAVARrO, M. y PARRA, F. (eds.). Sociología industrial y de la empresa. Madrid: Aguilar.

- (1996). Sociología del trabajo: Un proyecto docente. Madrid: Centro de Investigaciones Sociológicas / Siglo XXI.

- (2000). «División del trabajo, cualificación, competencias (Una guía para el análisis de formación por los trabajadores)", en colaboración con SANTOS, M.; GALAN, A.; Bono, A. del; Alas-Pumariño, A. Sociología del Trabajo, 40, 3-50.

CASTillo MendozA, C. y Terrén, E. (1994). «De la cualificación a la competencia: Elementos para una construcción epistemológica». Cuadernos de Relaciones Laborales, 4, 75-90.

COCKBURN, C. (1985). Machinery of dominance: women, men and technical know-how. Londres: Pluto Press.

Colardyn, D. (1996). La gestion des compétences: Perspectives internationales. París: Presses Universitaires de France.

CORIAT, B. (1993). El taller y el robot. Ensayos sobre el fordismo y la producción en masa en la era de la electrónica. Madrid: Siglo XX.

DERBER, CH. (1982). "The proletariarization of the professional: A Review Essay». En: Derber, CH. (ed). Professional as Workers: Mental Labor in Advanced Capitalism. Boston, Massachussets: G.K. Hall.

Evans, J. (1982). «El trabajador y el puesto de trabajo». En: FriedriCH, Gunter y SCHAFF, Adam (comps.). Microelectrónica y sociedad para bien o para mal. Madrid: Alhambra.

FinKel, L. (1994). La organización social del trabajo. Madrid: Pirámide. 
Freyssenet, M. (1979). «¿Es posible una definición única de cualificación?». Sociología del Trabajo, 2, 53-64.

GALLART, M. y JACINTO, C. (1996). «Adolescencia, pobreza, educación y formación para el trabajo». En: KOTERlLniK, I. y JACINTO, C. (comps.). Adolescencia, pobreza, educación y trabajo. Buenos Aires: UNICEF / Red Latinoamericana de Educación y Trabajo / Losada.

Haro, J. de (2004). "¿Sabe alguien qué es una competencia?». Dirigir Personas, 30, $8-17$.

Inda-Caro, M.; Rodríguez-Menéndez, C. y PeÑa-Calvo, V. (2010). «PISA 2006: La influencia del género en los conocimientos y competencias científicas». Revista Iberoamericana de Educación, 51/2, 1-12.

LAWLER, E. (1994). «From Job-Based to Competence-Based Organizations». Journal of Organizational Behavior, 15 (1), 3-15.

LE BOTERF, G. (2000). Ingeniería de las competencias. Barcelona: Gestión 2000.

Le Boterf, G.; Vincent, F. y Barzucchetti, S. (1993). Cómo gestionar la calidad de la formación. Barcelona: Gestión 2000.

Leborgne, D. y Lipietz, A. (1988). "L'après fordisme et son espace». Les Temps Modernes, 501, 75-114.

«Ley Orgánica 5/2002, de 19 de junio, de las cualificaciones y de la formación profesional». Boletin Oficial del Estado, 147 (20 de junio de 2002), 22437.

LITTLER, C. (1982). The development of the labour process in capitalist societies: a comparative study of the transformation of work organization in Britain, Japan and the USA. Londres; Exeter, N.H.: Heinemann Educational.

Lope, A. (1996). Innovación, tecnología y cualificación: La polarización de las cualificaciones en la empresa. Madrid: Consejo Económico y Social de España.

Mato, J. (1995). «¿Existe sobre-cualificación en España? Algunas variables explicativas». Documentos de Trabajo. Doc. 090/095. Oviedo: Facultad de Ciencias Económicas y Empresariales.

MCCLELland, D. (1973). "Testing for competence rather than for intelligence». American Psychologist, 28 (1), 13-20.

MCDERment, W. (1985). "Organisation du travail et formation professionnelle». Formation Professionnelle, 18, 66-76.

Milkman, R. y PUllman, C. (1991). «Technological change in an auto assembly part: the impact on workers' task and skills». Work and Occupations, 18 (2), 123-147.

Olaz, A. (2008). «Polarización Formativa en las Organizaciones. Análisis de Datos desde la Perspectiva del Darwinismo Social”. Praxis Sociológica, 12, 84 - 98.

- (2009). "Definición de un Modelo de Clima Laboral basado en la Gestión por Competencias». Papers, 93, 193-201.

PARra, E. y MORENO, F. (1993). "Clasificación profesional y movilidad». Economía y Sociología del Trabajo, 21-22, 169-175.

Pereda, S. y Berrocal, F. (2001). Gestión de recursos humanos por competencias. Madrid: Centro de Estudios Ramón Areces.

Petroni, G. (1987). «Nuove forme dei organizaciones del lavoro e mutamenti qualitativi dell tipologie professionali». En: Galantino, L. (comp.). Innovazione tecnológica e profesionalita del lavoratore. Padua: Cedam.

Piore, M. (1973). "The importance of human capital theory to labor economics: A dissenting View». Proceedings of the twenty-sixth annual winter meeting, Industrial Relations Research Association. Madison, Wis.: Industrial Relations Research Association, 251-258. 
Penn, R. y SCATtergood, R. (1978). Skilled manual workers in the labour process. Nuffield Paper.

PRIES, L. (1988). «Calificación, relaciones laborales y mercado de trabajo: el concepto de estrechez del ámbito empresarial en España». Revista de Investigaciones Sociológicas, 41, 81-114.

«Real Decreto 1128/2003, de 5 de septiembre, del catálogo nacional de cualificaciones profesionales». Boletín Oficial del Estado, 223 (17 de septiembre de 2003), 34293.

«Real Decreto 1312/2007, de 5 de octubre, de la acreditación nacional para el acceso a los cuerpos docentes universitarios». Boletín Oficial del Estado, 240 (17 de septiembre de 2003), 40653.

Roquero, E. y HeRnANDO, S. (2004). «La conformación del sistema nacional de cualificaciones profesionales en España: un proceso inacabado». Cuaderno de Relaciones Laborales, 22, 113-146.

SABEL, CH. (1986). Trabajo y política: la división del trabajo en la industria. Madrid: Ministerio de Trabajo y Seguridad Social.

SchulTz, TH. (1961). "Investment in human capital». American Economic Review, $51,1-17$.

Sorge, A. y STREECK, W. (1993). «Relaciones industriales y cambio técnico: Una perspectiva más amplia». En: HyMAN, R. y STREECK, W. (eds.). Nuevas tecnologías y relaciones industriales. Madrid: Ministerio de Trabajo.

SPENNER, K. (1985). "The upgrading and downgrading of occupations: issues, evidence and implications for education». Review of Educational Research, 55 (2), 125-154.

STROOBANTS, M. (1991). «Travail et competences: recapitulation critique des approches des savoirs au travail». Formation / Emploi, 33, 31-42.

- (1999). «Autour des mots gestion et competence». Recherche et Formation, 30, 61-69.

TuxworTh, E. (1989). "Competence Based Education and Training: Background and Origins». En: BURKE. J. (ed.). Competency based education and training. Londres: The Falmer Press.

Villavicencio, D. (1992). Por una definición de la calificación de los trabajadores. IV Congreso Español de Sociología (comunicación). Madrid.

WoOD, S. (1989). The transformation of work? Londres: Unwin Iman.

ZABALZA, M. (2007). Competencias docentes el profesorado universitario: calidad y desarrollo profesional. Madrid: Narcea. 